\title{
A Novel Composite Endpoint to Evaluate the Gastrointestinal Effects of NSAID Through the Entire GI Tract: Introducing CSULGIE
}

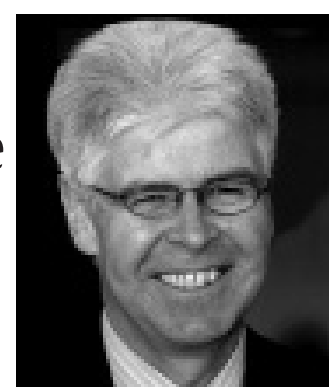

The notion that nonsteroidal antiinflammatory drugs (NSAID) cause gastric and duodenal ulcers, which can bleed or perforate, has certainly had its fair share of attention over the past 40 years. Yet, in rodent models, the burden of injury is in the terminal ileum, not the stomach or duodenum ${ }^{1}$. A small but growing constituency has argued that small bowel injury may be as important in humans. This proposition, which is commercially attractive to manufacturers of selective cyclooxygenase (COX)-2 inhibitors, which may lack this property, is increasingly discussed.

\section{Introducing CSULGIE}

In this issue of The Journal Francis Chan and his colleagues introduce the notion of Clinically Significant Upper and Lower GastroIntestinal Events ${ }^{2}$ (the acronym CSULGIE proudly maintaining the dysphonia originally established with the acronym NSAID!). They suggest that upper and lower GI events should be aggregated in assessing patients with drug-induced GI damage. In assessing this proposition, it is worthwhile reviewing lessons from the upper GI (UGI) tract.

\section{Lessons from the UGI Tract}

The first evidence that aspirin was gastrotoxic was conveyed by watercolor paintings of acute lesions detected by rigid endoscopy ${ }^{3}$, but the first systematic investigation used microbleeding as a surrogate for injury ${ }^{4}$ before volunteer endoscopy took over. Such studies also showed that non-aspirin NSAID caused injury, and numerous epidemiological studies associated aspirin and non-aspirin NSAID with GI bleeding 5,6. While endoscopy and a couple of secondary prevention studies were regarded as adequate to establish the validity of Helicobacter pylori eradication, different standards were applied to NSAID and COX-2 inhibitors with the advent of the outcomes study ${ }^{7}$.

\section{Acute or Chronic Lesions or Outcomes?}

As with $H$. pylori, endoscopy studies are broadly predictive of outcomes. Where there are differences, this may well be because of imprecisions in outcome studies. Nevertheless, the vogue for outcomes shows no signs of abating and accidentally generates interesting data and controversies along the way ${ }^{8}$.

\section{Endpoint Consensus}

Assessment of endoscopic endpoints developed in a somewhat haphazard way, and there has not always been validated consensus. The clearest example relates to acute studies of up to one month. All investigators sign up to similar definitions of an ulcer, but with a 10 -fold difference in estimates of acute ulcer formation.

\section{Lumping or Splitting?}

In older and newer trials of drugs for idiopathic ulcers or eradication of the $H$. pylori organism later found to cause them, gastric and duodenal ulcers were considered separately. For some reason in NSAID studies there was a tendency to lump them together. This has inhibited understanding, since NSAID-associated gastric duodenal ulcers differ in their association with $H$. pylori and their response to proton-pump inhibitors, $\mathrm{H} 2$ antagonists, and prostaglandin analogs 9,10 .

\section{Importance of Hypotheses}

To do a good outcomes study it is important to have a clear hypothesis, which may vary by mechanism. NSAID cause upper GI ulcer bleeding primarily because they cause upper GI ulcers, although there may be an additional antihemostatic effect with some NSAID, such as naproxen. With aspirin also, the balance may well be different, with the antihemostatic effect possibly dominating the ulcerogenic one. With naproxen both ulcerogenic and antihemostatic mechanisms may well apply.

\section{Importance of Precision}

Experience shows that the more exacting the endpoint definition, the more likely the hypothesis about it is to be

See A novel composite endpoint to evaluate the GI effects of NSAID, page 167 
proved. Most outcome studies have found COX-2 inhibitor use to result in around a halving of events ${ }^{8}$, a rather smaller reduction than would have been predicted if such drugs had placebo levels of toxicity. These studies have not exclusively measured ulcer complications but rather complicated events with or without uncomplicated ulcers. NSAID cause ulcers, so we would expect ulcer complications rather than any more general category of complicated events to be associated with NSAID and reduced by protective measures. The only study to test the hypothesis that ulcer complications would be reduced by use of a COX-2 inhibitor was the TARGET study of lumiracoxib ${ }^{11}$, which examined over 11 events against strict detailed criteria and showed upper GI ulcer complications to be reduced on lumiracoxib by $79 \%$ compared to NSAID, compatible with placebo levels of injury on lumiracoxib.

\section{Applying the Lessons from Upper GI to Lower GI Studies}

With lower GI lesions there has been a parallel progression to that seen with upper GI studies. The first human studies to suggest lower GI injury with NSAID employed indirect measures of damage including permeability changes and scanning of labelled neutrophils ${ }^{12}$. Capsule endoscopy supported these findings by associating mucosal erosions with NSAID $^{13,14}$ and showing a lower incidence with COX-2 inhibitors, while epidemiological studies and the results of some (but not most) outcome studies ${ }^{15}$ pointed to a high rate of complicated lower GI events on NSAID that fell with COX-2 inhibitors.

\section{Acute or Chronic Lesions or Outcomes?}

It is much less easy to form a hypothesis about the effects of NSAID on the lower GI tract. We know that NSAID-associated erosions and ulcers form principally in the small intestine, but many of the complicated events seen with NSAID, such as diverticular bleeding and perforation, occur from a different lesion at a different site. Knowledge is not yet sufficiently mature to make a judgment about whether these are related or distinct events.

\section{Endpoint Consensus}

Just as there is wide center to center fluctuation in acute upper GI studies, so too the number of erosions seen at capsule endoscopy varies significantly between centers. An international consensus based on blinded intraobserver variability studies is badly needed.

\section{Lumping or Splitting?}

The term CSULGIE favors lumping, but this has not served upper GI investigation very well. I would suggest it is premature to assume similar mechanisms for small-bowel erosions and diverticular bleeding and better at present to investigate these lesions separately.

\section{Importance of Precision}

Moreover, before we lump I think we should define much more clearly what the lesions of interest in the lower GI tract are. Are erosions detected by capsule important or trivial? Are they of similar significance in the ileum and jejunum? Which are the life-threatening complications of interest? In the VIGOR study 6 patients had diverticula. Other lesions were colonic ulcer, colitis, vascular ectasia, hemorrhoids, bleeding polyp, and rectal tear, none of which were seen in more than 2 patients. Might we not be best to treat diverticular complications, inflammatory bowel disease, and small-bowel lesions as separate pathologies?

\section{Importance of Hypotheses}

The only outcome study to show a difference between a COX-2 inhibitor and an NSAID in acute overt lower GI events was the VIGOR study, which compared rofecoxib with naproxen, a non-aspirin NSAID with uniquely potent antihemostatic properties. Given the negative findings in studies that compared with NSAID other than naproxen, the most likely explanation for differences in VIGOR surely relates to hemostasis, rather than COX-2 selectivity.

\section{Functional Consequence}

Chan and colleagues say that CSULGIE encompasses events in the lower GI tract such as clinically significant anemia. I disagree that this has been established as a "lower GI tract event." COX-2 inhibitors have been shown to both reduce small intestinal microulcers and result in a lower level of anemia, but no causal link between these 2 events has ever been established or any individual correlation reported. Indeed, to my knowledge, no differences in iron or ferritin levels have been shown, which would be necessary for the hypothesis to have even theoretical plausibility. It remains possible that the anemia on NSAID is due to dilutional fluid retention or a suppressive effect on the bone marrow of NSAID mucosal injury.

\section{Future Questions}

This is an area that needs investment in research to determine whether it is trivial phenomenology or important clinically. A number of studies could profitably be explored to address the following questions:

1. Is intestinal injury by NSAID a matter of COX inhibition or do other mechanisms predominate? These questions have been posed by Bjarnason and colleagues ${ }^{12}$ but much remains to be discovered.

2 . Is the extent to which the enterohepatic circulation contributes to lesion development in humans, as it seems to in animals, and therefore whether the morphology, significance, and natural history of terminal ileal lesions differ from more proximal ones?

3. Given that timing of intake in relation to food is a major determinant of ulceration and its distribution, 
should the validity of advice to take NSAID with food be evaluated?

4. Is the assumption that blood loss (as opposed to any other cause) from the small bowel (as opposed to elsewhere) causes anemia in patients on NSAID correct?

5. Are differences in overt bleeding between $\mathrm{COX}-2$ inhibitors and NSAID (where shown, as in the VIGOR study) simply due to differences in hemostasis?

6. Is the unproven assumption that NSAID provoke relapse of inflammatory bowel disease correct? There is precious little evidence that this is the case for Crohn's disease. In colitis there is as much evidence that paracetamol is associated with relapse as NSAID. Indeed, it was paracetamol that drove the initial differences that led to interest in this topic. 7. If paracetamol also causes relapse of colitis, what is the mechanism? Might an effect on the multi-drug MRP efflux system be implicated?

8. Is it just a subgroup of patients who are sensitive to paracetamol and NSAID?

\section{Research Sponsorship}

The authors acknowledge that their paper is based on a Pfizer publication. There is no reason to believe that this necessarily introduces a bias in the assessment. However, it is also surprising that this Pfizer-sponsored publication mentions all the ongoing Pfizer-funded studies except one, the SCOT study, which is currently recruiting about 200 patients per month and is on course to meet its trial objectives in 2012. This study uses the more exacting criteria of the TARGET study and provides an alternative assessment against which the value of CSULGIE could be tested.

CHRIS J. HAWKEY, DM, FRCP,

Professor of Gastroenterology, Nottingham University

University Hospital,

Nottingham, NG7 1AW United Kingdom

Address correspondence to Prof. Hawkey;

E-mail:cj.hawkey@nottingham.ac.uk

\section{REFERENCES}

1. Hawkey CJ. Nonsteroidal anti-inflammatory drug gastropathy. Gastroenterology 2000;119:521-35.

2. Chan FKL, Cryer B, Goldstein JL, Lanas A, Peura J, Scheiman JM, et al. A novel composite endpoint to evaluate the gastrointestinal (GI) effects of nonsteroidal antiinflammatory drugs through the entire GI tract. J Rheumatol 2010;37:167-74.
3. Douthwaite AH, Lintott GAM. Gastroscopic observation of the effect of aspirin and certain other substances on the stomach. Lancet 1938;232:1222-4.

4. Hunt JN, Franz D. Effect of prostaglandin E2 on gastric mucosal bleeding caused by aspirin. Dig Dis Sci 1981;26:301-5.

5. Henry D, Lim LL, Garcia Rodriguez LA, Perez Gutthann S, Carson $\mathrm{JL}$, Griffin M, et al. Variability in risk of gastrointestinal complications with individual non-steroidal anti-inflammatory drugs: results of a collaborative meta-analysis. BMJ 1996;312:1563-6.

6. Hawkey CJ. NSAIDs and aspirin: notorious or famous? Lancet 2009;374:93-4.

7. Hawkey CJ. Outcomes studies of drug induced ulcer complications. Do we need them and how should they be done? BMJ 2000;321:291-3.

8. Bombardier C, Laine L, Reicin A, Shapiro PH, Burgos-Vargas R, Davis B, et al. Comparison of upper gastrointestinal toxicity of rofecoxib and naproxen in patients with rheumatoid arthritis. N Eng J Med 2000;343:1520-8.

9. Hawkey CJ, Wilson I, Naesdal J, Langstrom G, Swannell AJ, Yeomans ND. Influence of sex and Helicobacter pylori on development and healing of gastroduodenal lesions in non-steroidal anti-inflammatory drug users. Gut 2002;51:344-50.

10. Hawkey CJ, Naesdal J, Wilson I, Langstrom G, Swannell AJ, Peacock RA, et al. Relative contribution of mucosal injury and Helicobacter pylori in the development of gastroduodenal lesions in patients taking non-steroidal anti-inflammatory drugs. Gut 2002;51:336-43.

11. Schnitzer TJ, Burmester GR, Mysler E, Hochberg MC, Doherty M, Ehrsam E, et al. Comparison of lumiracoxib with naproxen and ibuprofen in the Therapeutic Arthritis Research \& Gastrointestinal Event Trial (TARGET), in ulcer complications: Randomised controlled trial. Lancet 2004;364:665-74.

12. Bjarnason I, Prouse P, Smith T, Gumpel MJ, Zanelli G, Smethurst $\mathrm{P}$, et al. Blood and protein loss via small-intestinal inflammation induced by non-steroidal anti-inflammatory drugs. Lancet 1987;330:711-4.

13. Goldstein JL, Eisen GM, Lewis B, Gralnek IM, Aisenberg J, Bhadra P, et al. Small bowel mucosal injury is reduced in healthy subjects treated with celecoxib compared with ibuprofen plus omeprazole, as assessed by video capsule endoscopy. Aliment Pharmacol Ther 2007;25:1211-22.

14. Hawkey CJ, Ell C, Simon B, Albert J, Keuchel M, McAlindon M, et al. Less small-bowel injury with lumiracoxib compared with naproxen plus omeprazole. Clin Gastroenterol Hepatol 2008; 6:536-44.

15. Laine L, Connors LG, Reicin A, Hawkey CJ, Burgos-Vargas R, Schnitzer TJ, et al. Serious lower gastrointestinal clinical events with nonselective NSAID or coxib use. Gastroenterology 2003;124:288-92.

J Rheumatol 2010;37:6-8; doi:10.3899/jrheum.091207 